\title{
Control of a remote system over network including delays and packet dropout
}

\author{
Alexandre Seuret ${ }^{*, 1}$ Jean-Pierre Richard ${ }^{* *}$ \\ * Control and Instrumentation Research Group, Department of \\ Engineering, University of Leicester, University Road, Leicester, \\ LE1 7RH, UK. as389@le.ac.uk \\ ** LAGIS CNRS UMR 8146 and INRIA Equipe-Projet ALIEN, \\ Ecole Centrale de Lille, BP 48, 59651 Villeneuve d'Ascq Cedex, France \\ jean-pierre.richard@ec-lille.fr
}

\begin{abstract}
This work concerns the observer-based control of a remote, Master-Slave system through the Internet network. This communication link introduces variable, asymmetric and unpredictable delays, as well as packet loss. The data-sampling effects are also taken into account, even in the aperiodic case. Whereas the existing strategies require additional buffers, allowing the delay to become constant, the present result uses the information as soon as received. The proposed Lyapunov-Krasovskii functionals and LMI algorithms provide controller and observer gains which ensure the asymptotic stability of the global closed loop. The maximum admissible number of successive packets dropouts is also computed. The last part of the paper provides a simulation, where the Slave is a second-order system.
\end{abstract}

Keywords: NCS (networked controlled system), delay system, sampled-data system, asymptotic stabilization, packet loss, remote observer, Lyapunov-Krasovskii functional, LMI.

\section{INTRODUCTION AND HYPOTHESES}

Internet technology appears as a natural and cheap way to ensure the communication link in remotely controlled systems. Today, the available Quality of Service may seem to be good enough for that kind of applications. However, such a communication link constitutes an additional dynamical system, which great influence on stability was already mentioned in the 60's by Ferrel [1965]. Indeed, several dynamics and perturbations (communication delay, real-time sampling, packet dropout) are unavoidably introduced and have to be taken into account during the design of the control loop (see Richard and Divoux [2007]).

Fridman et al. [2004], Seuret et al. [2006] have shown that both sampling effects and communication time lags can be regrouped into an homogenized representation with timevarying delays. The present study also includes the packet loss effect into this unifying model. Once the global system is reduced to a system with delays, several control techniques can be involved (see for instance Niculescu [2001], Richard [2003]). Here, an observer-based instantaneous state feedback will be used. But, before presenting the details, a short overview of previous results will highlight the various assumptions on the communication delays $h_{1}(t)$ (from Master to Slave) and $h_{2}(t)$ (from Slave to Master).

Several works on tele-operation introduced the question of transmission delays in the constant case: Azorin et al. [2003], Fattouh and Sename [2003], Niemeyer and Slotine [1998]. However, in networked control situations, the delays are basically variable (jitter phenomenon). This is

1 This work was supported by an EPSRC Platform Grant reference EP/D029937/1 entitled 'Control of Complex Systems'. a source of problem when the classical predictor-based controllers are intended to be applied, since they generally need the delays to be constant, i.e. $h_{i}(t)=h_{i}$.

In the case of variable delays, some researches have used independent-of-delay conditions. Because such i.o.d. conditions may be conservative in general, Eusebi and Melchiorri [1998] considered particular cases such as constant or symmetric delays. This last assumption refers to the case where the transmission delays are equal, i.e. $h_{1}(t)=h_{2}(t)=R(t) / 2$, where $R(t)$ denotes the round trip time (RTT). In Garcia et al. [2000], non-symmetric delays were considered, but only in the constant delay case, i.e. $h_{1}(t)=h_{1} \neq h_{2}(t)=h_{2}$.

Another interesting approach was recently given by Witrant et al. [2007], which generalized the predictor techniques to the case of variable delays. In this case, a maximal bound of the delays is assumed to be known $\left(h_{M}\right.$ such that $\left.0 \leq h(t) \leq h_{M}\right)$, which is not that restrictive. The most constraining assumption is that an ordinary differential equation (ODE) model of the delay is supposed to be available, which is possible in the case of a singleowner network.

When using Internet, the generated delays are not only time-varying and non-symmetric, but also unknown (no dynamical model of the delays is available, see Niculescu et al. [2003]). To bypass this problem, it was proposed by Lelevé et al. [1999, 2001], N.J. Ploplys and Alleyne [2004] to introduce two input buffers (Master and Slave) that make both of the receivers wait until the maximum value $h_{M}$ of the communicaton delay is reached. However, it is obvious that this situation maximizes the delays $h_{1}(t)$ and 
$h_{2}(t)$ up to their worst (largest) value (i.e., $h_{1}(t) \hookrightarrow h_{M}$ and $\left.h_{2}(t) \hookrightarrow h_{M}\right)$ and, consequently, decreases the possible range of speed performance. To reduce this maximizing effect, Seuret et al. [2006, Sept., 2006] restricted the buffer to the only transmission from Master to Slave (thus, $\left.h_{1}(t) \hookrightarrow h_{M}\right)$, while the remote observer was computing the present Slave's state on the basis of the non-buffered Slave's output. The stabilizing gains were designed via some Lyapunov-Krasovskii functionals and, moreover, a guaranteed speed rate was computed. A first original contribution of the present study is to get rid of any buffer. We only assume the (non-symmetric) delays to have known minimal and maximal bounds $h_{m}$ and $h_{M}$, so that the following assumption holds:

$$
\text { A1 (maximal allowed delay): } h_{m} \leq h_{i}(t) \leq h_{M} \text {. }
$$

Since we aim at limiting the value of $h_{M}$, the use of UDP (User Datagram Protocol) is preferred to TCP (Transmission Control Protocol), the reliability mechanisms of which may needlessly slow down the feedback loop. In return, some data packets can be lost during the transmission, without being re-emitted. Such losses may affect the stabilization process. Taking the packet dropout into consideration constitutes a second contribution of this work since it was not taken into account in Seuret et al. [2006, Sept., 2006]. We take the following evaluation:

\section{A2 : Both Slave and Master receive at least one packet over $N$ consecutive.}

The proposed method will allow for computing some admissible (in the sense: non destabilizing) value of $N$.

An other feature of Internet is that the packets are not arriving in their chronological emission order, while UDP does not automatically re-organize them. Then, the reception function of Master and Slave will be added a reordering mechanism, based on some "time-stamps" added in the control and measurements packets. This mechanism ensures that the transmission delay variations satisfy:

$$
\text { A3 (packet reordering) : } \dot{h}_{i}(t)<1 \text {. }
$$

The last disturbances implied by the network come from the samplers and zero-holders needed for the discretetime implementation. Following the lines of Fridman et al. [2004], we consider they produce an additional variable delay $t-t_{k}, t_{k}$ being the $k^{t h}$ sampling instant. Moreover, because of the computer architecture and operating system, the sampling is generally not periodic, i.e. there is no period $T$ such that $t_{k}=k T$. So, we only assume there exists a known maximum sampling interval $T$ such that:

$$
\text { A4 (max. sampling interval) : } 0 \leq t_{k+1}-t_{k} \leq T \text {. }
$$

The global delays resulting from the communication-plussampling phenomena will be denoted by $\delta_{i}\left(t_{k}\right)=h_{i}(t)+$ $t-t_{k}$, for which the condition $\dot{\delta}_{i}(t) \leq 1$ holds. Note that the limit case $\dot{\delta}_{i}=1$ occurs.

As in Seuret et al. [2006], both the Slave and the Master are assumed to share a synchronized clock. This can be achieved via an additional GPS card or thanks to some tuning procedure (see Abdallah [2007]). As said before, the control and measurement packets are sent together with time-stamps, from which the receiver (Master or Slave) can deduce the non-symmetric delay value. By this way, both M-to-S $h_{1}(t)$ and S-to-M $h_{2}(t)$ delays are separately reconstructed by the system, and not only the RTT.

The paper is organized as follows: Section 2 describes the remote system features. Section 3 considers the problem of robust stability with respect to packet loss. Section 4 gives an illustrative example and Section 5, some concluding remarks.

\section{FEATURES OF THE REMOTE SYSTEM}

The exchanged data correspond to the control (sent by the Master to the Slave) and to the output of the remote system (sent by the Slave to the Master). The Slave is not supposed to have a large computation power and its functions are limited to: Receive control packets; Apply control; Send output measurement data. Thus the control and observation complexity is to be concentrated in the Master which has to: Receive output measurements; Estimate present state of Slave; Compute and Send the control value. Our purpose is to guarantee the asymptotic stability of the global Master-Slave system. In particular, the global system must ensure the closed-loop stability whatever the delay variation, the packet loss and the possible aperiodicity of the real-time sampling processes.

Stabilizing a system in such conditions is not easy. The Master receives the information he needs for the control computation after it has crossed the communication zone. The synchronism-based estimation of the transmission delay, joined to the observer, allows the Slave state to be known at the instant the information was sent to the Master. Similarly, the control computed by the Master will be applied some time after it is sent to the Slave, and this dead-time is not known in advance.

For the sake of simplicity, the Slave is considered to fit a linearized model. The stability must be robust with respect to the resulting, global delay. This property will be proven by using adequate Lyapunov-Krasovskii functionals, leading to an LMI optimization of the controller and observer gains. The system has the following features:

- The Master computes and forwards the control to the Slave. The forwarding induces a time-varying delay $h_{1}(t)$, assumed to satisfy $\mathbf{A} \mathbf{1}$ and $\mathbf{A} \mathbf{3}$, with packet loss for which A2 holds, as well as sampling effects which create the variable delay $\tau_{1}(t)$ satisfying $\mathbf{A 4}$.

- The Slave is driven by a controllable and observable, known model $(A, B, C)$, influenced by an input delay $\delta_{1}(t)$ to be defined later on in subsection 2.4:

$$
\left\{\begin{array}{l}
\dot{x}(t)=A x(t)+B u\left(t-\delta_{1}(t)\right) \\
y(t)=C x(t)
\end{array}\right.
$$

- The Slave measures its sampled output variables $y$, that the Master receives after a delay $h_{2}(t)$ which is also assumed to satisfy A1 (this assumption is not restrictive since it is obtained by the union of the intervals of variation of $h_{1}$ and $h_{2}$ ). The delay $\tau_{2}(t)$ due to the sampling is added. It means that the Master only can access $y\left(t-\delta_{2}(t)\right)$, where $\delta_{2}$ corresponds to the resulting delay. The Master includes an observer computing an estimate $\hat{x}$ of the complete Slave state $x$ at the present time $t$. From $\hat{x}$, the Master elaborates the control law (here, $u=K \hat{x}$ ). 
- The sampling instants $t_{k}$ may not be periodical i.e. $t_{k} \neq k T$, but it is supposed there exists a known $T$ such that A4 holds for any $k$.

- Both Master and Slave subsystems are synchronized, i.e. they share a same clock. Each data packet includes a time-stamp indicating the time the packet was sent. By this way, the receiver can calculate the transfer delays $h_{i}(t)$ as soon as it receives the packet.

The next subsections detail the features and notations.

\subsection{The sampling delays}

The sample $g\left(t_{k}\right)$ of a function $g(t)$ at time $t_{k}$ can be written as $g\left(t_{k}\right)=g\left(t-\left[t-t_{k}\right]\right)=g(t-\tau(t))$. This notation replaces the sample-and-hold with an additional delay $\tau_{k}(t)=t-t_{k}, t \in\left[t_{k}, t_{k+1}[\right.$. Thus, under assumption A4 the aperiodic sampling is modeled as an unknown delay $\tau(t) \leq T$. This change allows continuous-time stability techniques (e.g. Lyapunov-Krasovskii functionals) to be used for sampled systems.

\subsection{The control law}

The controller computes a control law which considers some set-values $x_{s}$ to be reached by the Slave. The static state feedback control $u(t)=K\left(\hat{x}(t)-x_{s}(t)\right)$ is defined considering the state estimate $\hat{x}$ given by the observer. The main difficulty is to determine the linear gain $K$ of the state feedback control in order to guarantee the asymptotic stability of the Slave motion despite the values of the (unknown, time-varying) control delays. Without being restrictive with regard to stability, we consider $x_{s}(t)=0$.

\subsection{Transmission of the control $u$}

The $k^{t h}$ packet sent by the Master to the Slave includes the designed control $u\left(t_{1, k}\right)$ and the instant of time $t_{1, k}$ when the packet was sent. The Slave receives this information at time $t_{1, k}^{r}$. Thanks to the clock synchronization, this time has the same meaning for both the Slave and the Master. Then, the term $t_{1, k}^{r}-t_{1, k}$, corresponding to the transmission delay, is known by the Slave once the packet has reached it.

\subsection{Receipt and processing of the control data}

The control, sent at time $t_{1, k}$, is received by the Slave at time $t_{1, k}^{r} \geq t_{1, k}+h_{m}$. There is no reason that the Master also knows the time $t_{1, k}^{r}$ when the control $u\left(t_{1, k}\right)$ will be injected into the Slave input.

\subsection{Transmission of the measured output information}

The Slave accesses its output $y$ at discrete instants of time. He sends packet containing the output $y\left(t_{2, k^{\prime}}\right)$ and the measurement instant $t_{2, k^{\prime}}$ which is the $k^{\prime t h}$ one. The Master receives the output data at time $t_{2, k^{\prime}}^{r}$. Once the packet has reached the Master, the delay $t_{2, k^{\prime}}^{r}-t_{2, k^{\prime}}$ is known due to the synchronization.

\subsection{Observation of the process}

For a given $\hat{k}$ and any $t \in\left[t_{1, \hat{k}}+\left(h_{M}-h_{m}\right) / 2, t_{1, k+1}+\right.$ $\left(h_{M}-h_{m}\right) / 2\left[\right.$, there exists a $k^{\prime}$ such that the proposed observer is of the form:

$$
\left\{\begin{array}{l}
\dot{\hat{x}}(t)=A \hat{x}(t)+B u\left(t_{1, \hat{k}}\right)-L\left(y\left(t_{2, k^{\prime}}\right)-\hat{y}\left(t_{2, k^{\prime}}\right)\right), \\
\hat{y}(t)=C \hat{x}(t) .
\end{array}\right.
$$

The time stamp $t_{1, \hat{k}}$ corresponds to the time when the control input is supposed to be injected in the Slave. The index $k^{\prime}$ corresponds to the most recent output information the Master has received. Note that the Master is not supposed to know the time $t_{1, k}^{r}$ and the control $u\left(t_{1, k}\right)$ (see Section 2.4), which makes this observer realizable. The input delay approach to sampled-data signals allows a homogenized definition of the delays $\delta_{1}(t) \triangleq t-t_{1, k}$ where $k$ corresponds to the real sampling implemented in the Slave process, $\hat{\delta}_{1}(t) \triangleq t-t_{1, \hat{k}}$ and $\delta_{2}(t) \triangleq t-t_{2, k^{\prime}}$ to be considered and the observer dynamics can be written:

$$
\left\{\begin{aligned}
\dot{\hat{x}}(t)= & A \hat{x}(t)+B u\left(t-\hat{\delta}_{1}(t)\right) \\
& -L\left(y\left(t-\delta_{2}(t)\right)-\hat{y}\left(t-\delta_{2}(t)\right)\right), \\
\hat{y}(t)= & C \hat{x}(t),
\end{aligned}\right.
$$

In other words, the observer is realizable since $t_{2, k^{\prime}}$ defining the observer delay is known due to the synchronization. In the ideal case where $N=1$ (from A2, no packet loss) and where the Master-to-Slave delay is assumed to be well known, i.e. $\delta_{1}(t)=\hat{\delta}_{1}(t)$ (obtained by means of a buffer in Seuret et al. [2006]), the global system can then be rewritten using the error vector $e(t)=x(t)-\hat{x}(t)$ as:

$$
\begin{aligned}
\dot{x}(t) & =A x(t)+B K x\left(t-\delta_{1}(t)\right)-B K e\left(t-\delta_{1}(t)\right), \\
\dot{e}(t) & =A e(t)+L C e\left(t-\delta_{2}(t)\right),
\end{aligned}
$$

where the features of the system lead, for $i=1,2$, to $h_{m} \leq \delta_{i}(t) \leq h_{M}+T$. Equivalently, if one uses the average delay $\delta\left(h_{m}, h_{M}, T\right)=\left(h_{M}+T+h_{m}\right) / 2$ and the maximum delay amplitude $\mu\left(h_{m}, h_{M}, T\right)=\left(h_{M}+T-h_{m}\right) / 2$, then:

$$
\delta-\mu \leq \delta_{i}(t) \leq \delta+\mu, \quad \forall i=1,2 .
$$

For this ideal case, Theorems 2 and 3 by Seuret et al. [2006] deliver controller and observer gains.

\section{STABILIZATION WITH PACKET LOSS}

This section focusses on a more realistic model of the remote system detailed in Fig. 1. It is now accepted that $\delta_{1}(t) \neq \hat{\delta}_{1}(t)$ and that data packets can be lost during the transfer between Master and Slave. Since the UDP protocol is used, packets dropouts are frequent. When a data-packet is lost during the transmission process, the delays $\delta_{i}(t)$ can be easily increased up to $\delta+\mu$ as defined in (9) and go greater. Then, the stability conditions from Theorems 2 and 3 in Seuret et al. [2006] do not ensure the stability of the global remote system anymore. Packet losses have another effect on the system. Since the observer uses the input control $u\left(t_{1, k}\right)$ which is supposed to be implemented in the slave process, if a control-data packet is lost, the observer will not use the same control. In compensation, the assumption $\mathbf{A 2}$ (with $N \geq 2$ ) is made on the packet loss phenomenon.

Given $k$ and any $t \in\left[t_{1, k}^{r}+h_{m}, t_{1, k+1}^{r}+h_{m}\right.$ [, there exist $\hat{k}$ and $k^{\prime}$ such that the proposed observer is of the form: 
Fig. 1. Structure of the remote system under time-varying delays, samplings and packet loss.

$$
\begin{aligned}
\dot{x}(t)= & A x(t)+B K \hat{x}\left(t_{1,\left(k-p_{1}\right)}\right), \\
\dot{\hat{x}}(t)= & A \hat{x}(t)+B K \hat{x}\left(t_{1, \hat{k}}\right) \\
& -L C\left(x\left(t_{2,\left(k^{\prime}-p_{2}\right)}\right)-\hat{x}\left(t_{2,\left(k^{\prime}-p_{2}\right)}\right) .\right.
\end{aligned}
$$

where the positive integers $p_{1} \leq N$ and $p_{2} \leq N$ represent the packet losses. Rewriting the equations by using the error $e(t)=x(t)-\hat{x}(t)$, the dynamics become:

$$
\begin{aligned}
\dot{x}(t)= & A x(t)+B K x\left(t_{1,\left(k-p_{1}\right)}\right)-B K e\left(t_{1,\left(k-p_{1}\right)}\right), \\
\dot{e}(t)= & A e(t)+\operatorname{LCe}\left(t_{2,\left(k^{\prime}-p_{2}\right)}\right) \\
& -B K \int_{t_{1,\left(k-p_{1}\right)}}^{t_{1, \hat{k}}}[\dot{x}(s)-\dot{e}(s)] d s .
\end{aligned}
$$

Applying the input delay representation Fridman et al. [2004] yields:

$$
\begin{aligned}
\dot{x}(t)= & A x(t)+B K x\left(t-\delta_{1}(t)\right)-B K e\left(t-\delta_{1}(t)\right), \\
\dot{e}(t)= & A e(t)+L C e\left(t-\delta_{2}(t)\right) \\
& -B K \int_{t_{1,\left(k-p_{1}\right)}}^{t_{1, \hat{k}}}[\dot{x}(s)-\dot{e}(s)] d s,
\end{aligned}
$$

with $\delta_{1}(t)=t-t_{1,\left(k-p_{1}\right)}$ and $\delta_{2}(t)=t-t_{2,\left(k^{\prime}-p_{2}\right)}$. From the fact that the communication delays belong to the interval $\left[h_{m}, h_{M}\right]$ where $h_{m}$ and $h_{M}$ are given by the network properties and by noting that the assumption on the packet loss leads to $t_{1, \hat{k}}-t_{1,\left(k-p_{1}\right)} \leq N T+\mu$ where $\mu$ is defined above, the inequalities $h_{m} \leq \delta_{i}(t) \leq h_{M}+N T$ hold for $i=1,2$. It is now possible to define the average delay $\delta\left(h_{m}, h_{M}, N, T\right)$ and the maximal delay amplitude $\mu\left(h_{m}, h_{M}, N, T\right)$ as:

$$
\begin{aligned}
& \delta\left(h_{m}, h_{M}, N, T\right)=\left(h_{M}+N T+h_{m}\right) / 2, \\
& \mu\left(h_{m}, h_{M}, N, T\right)=\left(h_{M}+N T-h_{m}\right) / 2
\end{aligned}
$$

For the sake of simplicity in the notation, only $\delta$ and $\mu$ will be used. Then, both of the resulting delays satisfy:

$$
\delta-\mu \leq \delta_{i}(t) \leq \delta+\mu, \quad i=1,2 .
$$

As in equation (11), there are interconnection terms between the two variables $x$ and $e$, a separation principle is no longer applicable to prove the global stabilization as it was suggested in Seuret et al. [Sept., 2006]. The stability proof requires to consider now both variables simultaneously.

Theorem 1. For given $K$ and $L$, for $q$ representing the subscript $x$ or $e$, suppose there exist positive definite matrices $P_{q 1}, S_{q}, R_{q a}, R_{q p}, S_{x e}$ and $R_{x e}$ and matrices of size $n \times n$ : $P_{q 2}, P_{q 3}, Z_{q l}$ for $l=1,2,3, Y_{q l^{\prime}}$ for $l^{\prime}=1,2$, such that the following LMI's hold:

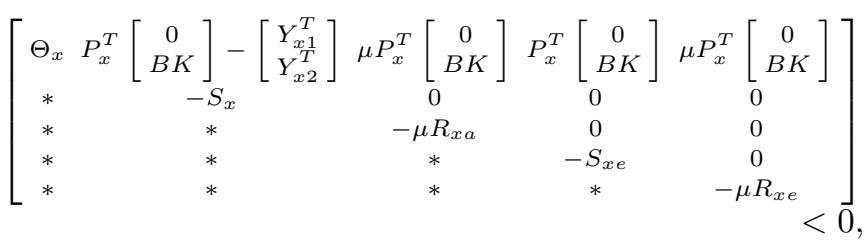

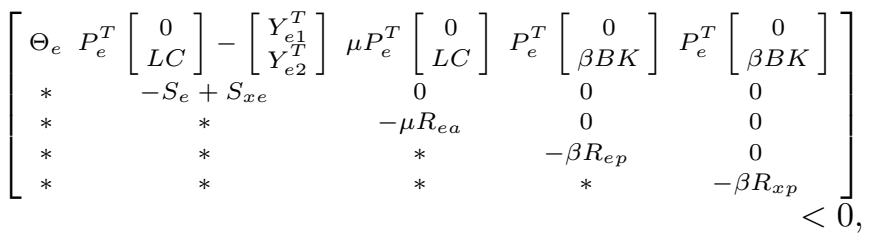

$$
\left[\begin{array}{ccc}
R_{q} & Y_{q 1} & Y_{q 2} \\
* & Z_{q 1} & Z_{q 2} \\
* & * & Z_{q 3}
\end{array}\right] \geq 0, \quad q \in\{x, e\}
$$

where $\beta=2 \mu, P_{q}=\left[\begin{array}{cc}P_{q 1} & 0 \\ P_{q 2} & P_{q 3}\end{array}\right]$, and

$$
\begin{aligned}
\Theta_{x}= & \Theta_{x}^{n}+\left[\begin{array}{cc}
0 & 0 \\
0 & 2 \mu R_{x p}
\end{array}\right], \Theta_{e}=\Theta_{e}^{n}+\left[\begin{array}{cc}
0 & 0 \\
0 & 2 \mu\left(R_{e p}+R_{x e}\right)
\end{array}\right], \\
\Theta_{q}^{n}= & P_{q}^{T}\left[\begin{array}{cc}
0 & I \\
A & -I
\end{array}\right]+\left[\begin{array}{cc}
0 & I \\
A & -I
\end{array}\right]^{T} P_{q} \\
& +\left[\begin{array}{cc}
S_{q}+Y_{q 1}+Y_{q 1}^{T}+\delta Z_{q 1} & Y_{q 2}+\delta Z_{q 2} \\
* & \delta R_{q}+2 \mu R_{q a}+\delta Z_{q 3}
\end{array}\right] .
\end{aligned}
$$

Then, the global remote system (10) is asymptotic stable.

Proof: The proof is given in the appendix.

Remark 1. Theorem 1 allows the robust stability of the global remote system to be guaranteed with respect to the packet dropout and for observer and controller gains given in Seuret et al. [2006]. Since the problems of designing observer and controller gains are dual, to develop constructive LMI's for obtaining both gains is not straightforward. Another solution would be to develop conditions in order to design the controller gain for a given observer gain and other conditions to solve the opposite problem.

\section{APPLICATION TO A MOBILE ROBOT}

This study is illustrated on the model of a mobile robot (Slave) moving in one direction. The identification phase gives the following dynamics:

$$
\left\{\begin{array}{l}
\dot{x}(t)=\left[\begin{array}{cc}
0 & 1 \\
0 & -11,32
\end{array}\right] x(t)+\left[\begin{array}{c}
0 \\
11,32
\end{array}\right] u\left(t-\delta_{1}(t)\right), \\
y(t)=\left[\begin{array}{ll}
1 & 0
\end{array}\right] x(t)
\end{array}\right.
$$

The characteristics of our network (Internet, M-S distance $=50 \mathrm{~km}$, France) allow for $h_{m}=0,1 \mathrm{~s}$ and $h_{M}=0.4 \mathrm{~s}$. Consider now that the bandwidth of the network allows the sampling period as $T=0.1 \mathrm{~s}$ to be defined. For these values, Theorems 2 and 3 in Seuret et al. [2006]

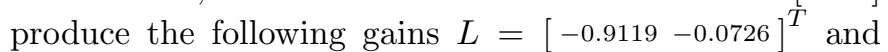
$K=\left[\begin{array}{ll}-0.9125 & -0.0801\end{array}\right]$. This gains ensures that, in the ideal case the remote system is $\alpha$-stable for $\alpha_{x}=\alpha_{e}=$ 1.05. Theorem 1 ensures that, with these features, the global system (8) is asymptotically stable and robust with respect to the packet loss for all $N \leq 12$ in (2). Moreover it guarantees asymptotic stability of the global system without the introduction of a buffer in the controller.

\section{CONCLUDING REMARKS}

This paper has proposed a new strategy for an observerbased control of a remote process. Various perturbations were dealt with: (1) jittery, non-symmetric and unpredictable delays (Internet); (2) packet dropouts (UDP) and (3) aperiodic sampling (real-time). No buffering technique was involved, which allows both the Master and the Slave to use the available information as soon as received. 
A characteristic feature of this control strategy is to consider that the Master runs in continuous time (i.e., with small computation step) whereas the Slave provides discrete-time measurements. Thus, the observer keeps on providing a continuous estimation of the present Slave state, even if the Slave information is not sent continuously. Technically speaking, it is also noticeable that the corresponding stability criterion considers non-small delays, i.e. delays which lower bound is non zero.

A remaining assumption is that Master and Slave's clocks are synchronized. This property is needed for the delay measurement and can be obtained via technical (GPS) or computational Abdallah [2007] solutions. However, the ongoing implementations of a related strategy Seuret et al. [2006, Sept., 2006] show that the global system may work even if the clocks are a bit out of synch. Including this robustness in the design is left to future works.

\section{APPENDIX: PROOF OF THEOREM 3}

To analyze the asymptotic stability property of such a system, equations (11) are rewritten by using the descriptor representation introduced in Fridman [2001], Fridman and Shaked [2002] with $\bar{x}(t)=\operatorname{col}\{x(t), \dot{x}(t)\}$, $\bar{e}(t)=\operatorname{col}\{e(t), \dot{e}(t)\}$. Consider the following LyapunovKrasovskii functional:

$V(t)=V_{x n}(t)+V_{x a}(t)+V_{x p}(t)+V_{e n}(t)+V_{e a}(t)+V_{e p}(t)$

where the sub-Lyapunov-Krasovskii functionals are, for $q$ representing the subscript of the variables ' $x$ ' and ' $e$ ':

$$
\begin{aligned}
V_{q n}(t)= & \bar{q}^{T}(t) E P_{q} \bar{q}(t)+\int_{-\delta}^{0} \int_{t+\theta}^{t} \dot{q}^{T}(s) R_{q} \dot{q}(s) d s d \theta \\
& +\int_{t-\delta}^{t} q^{T}(s) S_{q} q(s) d s, \\
V_{q a}(t)= & \int_{-\mu}^{\mu} \int_{t+\theta-\delta}^{t} \dot{q}^{T}(s) R_{q a} \dot{q}(s) d s d \theta, \\
V_{x p}(t)= & \int_{-\mu}^{\mu} \int_{t+\theta-\delta}^{t} \dot{x}^{T}(s) R_{x p} \dot{x}(s) d s d \theta, \\
V_{e p}(t)= & \int_{-\mu}^{\mu} \int_{t+\theta-\delta}^{t} \dot{e}^{T}(s)\left(R_{e p}+R_{x e}\right) \dot{e}(s) d s d \theta,
\end{aligned}
$$

with $E=\operatorname{diag}\left\{I_{n}, 0\right\}$ and $P_{x}, P_{e}$ defined in Theorem 1 .

The signification of each sub-Lyapunov-Krasovskii functional has to be explain. The first functionals $V_{x n}$ and $V_{\text {en }}$ deal with the stability of the Slave and the observer systems subject to the constant delay $\delta$ while $V_{x a}$ and $V_{e a}$ refer to the disturbances due to the delay variations. Even if the functionals do not explicitly depend on each time varying delay, it will be considered two different delays $\delta_{1}$ and $\delta_{2}$. The last functionals $V_{x p}$ and $V_{e p}$ are concerned with the packet loss. This will appear more clearly later on. According to Theorem 2 in Seuret et al. [2005], if LMI (16) holds for ' $q=x^{\prime}$, the following inequalities hold:

$$
\dot{V}_{x n}(t)+\dot{V}_{x a}(t) \leq \xi_{x}^{T}(t)\left[\begin{array}{cc}
\Psi_{x 1} & P_{x}^{T}\left[\begin{array}{c}
0 \\
B K
\end{array}\right] \\
* & -S_{x}
\end{array}\right] \xi_{x}(t)+\eta_{x}(t),
$$

where $\xi_{x}(t)=\operatorname{col}\{x(t), \dot{x}(t), x(t-\delta)\}$ and:

$$
\begin{aligned}
& \eta_{x}(t)=-2 \bar{x}^{T}(t) P_{x}^{T}\left[0(B K)^{T}\right] e\left(t-\delta_{1}(t)\right), \\
& \Psi_{x 1}=\Theta_{x}^{n}+\mu P_{x}^{T}\left[0(B K)^{T}\right]^{T} R_{x a}^{-1}\left[0(B K)^{T}\right] P_{x},
\end{aligned}
$$

Noting that $e\left(t-\delta_{1}(t)\right)=e(t-\delta)-\int_{t-\delta_{1}(t)}^{t-\delta} \dot{e}(s) d s$ and using a classical LMI bounding, the following inequality holds for $i=1,2$ :

$$
\begin{aligned}
\eta_{x}(t) & \leq \bar{x}^{T}(t) P_{x}^{T}\left[\begin{array}{c}
0 \\
B K
\end{array}\right]\left(S_{x e}^{-1}+\mu R_{x e}^{-1}\right)\left[\begin{array}{c}
0 \\
B K
\end{array}\right]^{T} P_{x} \bar{x}(t) \\
& +e^{T}(t-\delta) S_{x e} e(t-\delta)+\left|\int_{t-\delta_{1}(t)}^{t-\delta} \dot{e}^{T}(s) R_{x e} \dot{e}(s) d s\right|
\end{aligned}
$$

where $S_{x e}$ and $R_{x e}$ are positive definite matrices which represent the presence of the error vector in the state equation. Then, the following inequality holds:

$$
\begin{aligned}
& \dot{V}_{x n}(t)+\dot{V}_{x a}(t) \leq \\
& \left.\quad \xi_{x}^{T}(t)\left[\begin{array}{cc}
\Psi_{x 2} & P_{x}^{T} \\
* & 0 \\
B K
\end{array}\right]-\left[\begin{array}{c}
Y_{x 1}^{T} \\
Y_{x 2}^{T}
\end{array}\right]\right] \xi_{x}(t) \\
& \quad+e^{T}(t-\delta) S_{x e} e(t-\delta)+\left|\int_{t-\delta_{1}(t)}^{t-\delta} \dot{e}^{T}(s) R_{x e} \dot{e}(s) d s\right|,
\end{aligned}
$$

where $\Psi_{x 2}=\Theta_{x}^{n}+P_{x}^{T}\left[0(B K)^{T}\right]^{T}\left(S_{x e}^{-1}+\mu R_{x a}^{-1}+\right.$ $\left.\mu R_{x e}^{-1}\right)[0(B K)] P_{x}$.

For the error dynamics, LMI (16) with $q=e$ yields:

$$
\begin{aligned}
\dot{V}_{e n}(t)+\dot{V}_{e a}(t) \leq & \xi_{e}^{T}(t)\left[\begin{array}{cc}
\Psi_{e 1} & P_{e}^{T}\left[\begin{array}{c}
0 \\
L C
\end{array}\right]-Y_{e}^{T} \\
* & -S_{e}
\end{array}\right] \xi_{e}(t) \\
& -\eta_{e}^{x}(t)+\eta_{e}^{e}(t)
\end{aligned}
$$

where $\xi_{e}(t)=\operatorname{col}\{e(t), \dot{e}(t), e(t-\delta)\}$ and where $\Psi_{e 1}=$ $\Theta_{e}^{n}+\mu P_{e}^{T}\left[0(L C)^{T}\right]^{T} R_{e a}^{-1}[0(L C)] P_{e}$, and $\eta_{e}^{q}(t)=2 \bar{e}^{T}(t)$ $\times P_{e}^{T}\left[0(B K)^{T}\right]^{T} \int_{t_{1,\left(k-p_{1}\right)}}^{t_{1, \hat{k}}} \dot{q}(s) d s$ with $q$ representing either $x$ or $e$. Note that the functions $\eta_{e}^{x}(t)$ and $\eta_{e}^{e}(t)$ correspond to the disturbance due to the lost control packets. The following equality holds:

$$
-\eta_{e}^{x}(t)=-2 \bar{e}^{T}(t) P_{e}^{T}\left[\begin{array}{c}
0 \\
B K
\end{array}\right] \int_{t_{1,\left(k-p_{1}\right)}}^{t_{1, \hat{k}}} \dot{x}(s) d s .
$$

Noting that from $\mathbf{A} 4$, one has $t_{1, \hat{k}}-t_{1,\left(k-p_{1}\right)} \leq 2 \mu$, thus:

$$
\begin{aligned}
\eta_{e}^{x}(t) \leq & 2 \mu \bar{e}^{T}(t) P_{e}^{T}\left[\begin{array}{c}
0 \\
B K
\end{array}\right] R_{x p}^{-1}\left[\begin{array}{c}
0 \\
B K
\end{array}\right]^{T} P_{e} \bar{e}(t) \\
& +\int_{t_{1,\left(k-p_{1}\right)}}^{t_{1, \hat{k}}} \dot{x}^{T}(s) R_{x p} \dot{x}(s) d s .
\end{aligned}
$$

In the same way, the following inequality holds:

$$
\begin{aligned}
\eta_{e}^{e}(t) \leq & 2 \mu \bar{e}^{T}(t) P_{e}^{T}\left[\begin{array}{c}
0 \\
B K
\end{array}\right] R_{e p}^{-1}\left[\begin{array}{c}
0 \\
B K
\end{array}\right]^{T} P_{e} \bar{e}(t) \\
& +\int_{t_{1,\left(k-p_{1}\right)}}^{t_{1, \hat{k}}} \dot{e}^{T}(s) R_{e p} \dot{e}(s) d s .
\end{aligned}
$$

Finally, the following inequality holds:

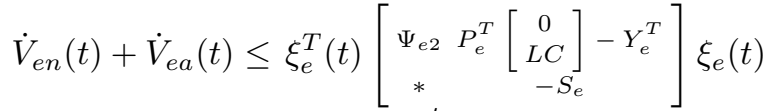

$$
\begin{aligned}
& +\sum_{q=x, e} \int_{t_{1,\left(k-p_{1}\right)}}^{t_{1, \hat{k}}} \dot{q}^{T}(s) R_{q p} \dot{q}(s) d s,
\end{aligned}
$$

where

$$
\begin{aligned}
\Psi_{e 2}= & \Theta_{e}^{n}+\mu P_{e}^{T}\left[\begin{array}{c}
0 \\
L C
\end{array}\right] R_{e a}^{-1}\left[\begin{array}{c}
0 \\
L C
\end{array}\right]^{T} P_{e} \\
& +2 \mu P_{e}^{T}\left[\begin{array}{c}
0 \\
B K
\end{array}\right]\left(R_{x p}^{-1}+R_{e p}^{-1}\right)\left[\begin{array}{c}
0 \\
B K
\end{array}\right]^{T} P_{e} .
\end{aligned}
$$

Differentiating $V_{x p}$ and $V_{e p}$ along the trajectories leads to inequalities (26) for the state vector and error dynamics: 


$$
\begin{aligned}
\dot{V}_{x p}(t)= & 2 \mu \dot{x}^{T}(t) R_{x p} \dot{x}(t) \\
& -\int_{t-\delta-\mu}^{t-\delta+\mu} \dot{x}^{T}(s) R_{x p} \dot{x}(s) d s \\
\dot{V}_{e p}(t)= & 2 \mu \dot{e}^{T}(t)\left(R_{e p}+R_{x e}\right) \dot{e}(t) \\
& -\int_{t-\delta-\mu}^{t-\delta+\mu} \dot{e}^{T}(s)\left(R_{e p}+R_{x e}\right) \dot{e}(s) d s .
\end{aligned}
$$

Combining (21), (25) and (26) and noting that the sum of the negative integrals in (26) with the integrals from (24) is negative because $t_{1, \hat{k}}$ and $t_{1,(k-p 1)}$ are included in the interval $[t-\delta-\mu, t-\delta+\mu]$, the following inequality holds:

$$
\begin{aligned}
& \left.\dot{V}(t) \leq \xi_{x}^{T}(t)\left[\begin{array}{cc}
\Psi_{x 2} & P_{x}^{T} \\
* & -S_{x} \\
B K
\end{array}\right]\right] \xi_{x}(t) \\
& +\xi_{e}^{T}(t)\left[\begin{array}{cc}
\Psi_{e 2} & P_{e}^{T}\left[\begin{array}{c}
0 \\
L C
\end{array}\right] \\
* & -S_{e}+S_{x e}
\end{array}\right] \xi_{e}(t) .
\end{aligned}
$$

The Schur complement leads to the LMI's given in (14) and (15). Then if LMI's from Theorem 1 are satisfied, the system (11) is asymptotically stable.

\section{REFERENCES}

C. T. Abdallah. Delay effect in the networked control of mobile robot. in "Application in Time-Delay Systems, Edt J. Chiasson and J.-J. Loiseau, LNCIS 352, Springer-Verlag, Berlin Heidelberg, 2007.

J.M. Azorin, O. Reinoso, J.M. Sabater, R.P. Neco, and R. Aracil. Dynamic analysis for a teleoperation system with time delay. In Conf. on Control Applications, June 2003.

A. Eusebi and C. Melchiorri. Force-reflecting telemanipulators with time-delay: Stability analysis and control design. IEEE trans. on Robotics and Automation, 14(4): 635-640, 1998.

A. Fattouh and O. Sename. $H^{\infty}$-based impedance control of teleoperation systems with time delay. In 4 th Workshop on Time Delay Systems, September 2003.

W.R. Ferrel. Remote manipulation with transmission delay. IEEE trans. Human Factors Electr., HFE:24-32, 1965.

E. Fridman. New Lyapunov-Krasovskii functionals for stability of linear retarded and neutral type systems. System and Control Letters, 43:309-319, 2001.

E. Fridman and U. Shaked. A descriptor system approach to $H^{\infty}$ control of linear time-delay systems. IEEE Trans. on Automatic Control, 47(2):253-270, 2002.

E. Fridman, A. Seuret, and J.-P. Richard. Robust sampleddata stabilization of linear systems: An input delay approach. Automatica, 40(8):1141-1446, 2004.

C.E. Garcia, B. Morales, R. Carelli, and J. Postigo. Stability analysis for a teleoperation system with time delay and force feedback. In Proceeding of the $39^{t h}$ Conference on Decision and Control, editors, Conference on Decision and Control, December 2000.

A. Lelevé, P. Fraisse, P. Dauchez, and F. Pierrot. Stability analysis for a teleoperation system with time delay and force feedback. In Proceedings. IEEE/ASME International Conference, December 1999.

A. Lelevé, P. Fraisse, and P. Dauchez. Telerobotics over ip networks: Towards a low-level real-time architecture. In Proceedings. IEEE/RSF International Conference, volume 2, Novembre 2001.
S.-I. Niculescu. Delay Effects on Stability. A Robust Control Approach. Springer-Verlag, 2001.

S.-I. Niculescu, C.T. Abdallah, and P.F. Hokayem. Effects of channel dynamics on the stability of teleoparation. In 4th Workshop on Time Delay Systems, September 2003.

G. Niemeyer and J.-J. Slotine. Towards force-reflecting teleoparation over the internet. In IEEE Int. Con. on Robotics \& Automation, 1998.

P.A. Kawka N.J. Ploplys and A.G. Alleyne. Closed-loop control over wireless networks. IEEE Control Systems Magazine, 24(3):58-71, June 2004.

J.-P. Richard. Time delay systems: an overview of some recent advances and open problems. Automatica, 39: 1667-1694, 2003.

J.P. Richard and T. Divoux. Systèmes commandés en réseau. Hermès-Lavoisier, 2007.

A. Seuret, E. Fridman, and J.-P. Richard. Sampleddata exponential stabilization of neutral systems with input and state delays. In IEEE MED 2005, $13^{\text {th }}$ Mediterranean Conference on Control and Automation, June 2005.

A. Seuret, F. Michaut, J.-P. Richard, and T. Divoux. Networked control using GPS synchronization. In American Control Conference, Minneapolis, US, June 2006.

A. Seuret, M. Termens-Balester, A. Toguyeni, S. El Khatabi, and J.-P. Richard. Implementation of an internet-controlled system under variable delays. In ETFA'06, Praha, Czech Republic, Sept., 2006.

E. Witrant, C. Canudas de Wit, D. Georges, and M. Alamir. Remote output stabilization via communication networks with a distributed control law. IEEE Trans. on Automatic Control, 52(8):1480-1485, 2007. 